\title{
Safer insulin needle use and disposal
}

\author{
Rebecca Furth ${ }^{1}$, Audrey Anderson ${ }^{1}$, Gumti Krishendat ${ }^{2}$ \\ ${ }^{1}$ Initiatives Inc., Boston, USA \\ ${ }^{2}$ Chronic Disease Unit, Guyana Ministry of Health, Guyana
}

doi: 10.3396/ijic.V6i2.012.10

\begin{abstract}
The safe use and disposal of injection equipment is a major component of infection control in clinical and community settings. Diabetic insulin users represent a significant population of injection equipment consumers whose practices have implications for community safety. Most insulin users inject at home; yet even in developed countries, there are few options for safely disposing of needles or lancets in the home setting. This paper presents the results of a pilot program designed to test out low cost, practical options for improving insulin syringe use and disposal safety in Guyana. The Pilot tested out the provision of a full monthly supply of insulin syringes to try and address re-use, trialed four different containers to contain used needles at home and return them to health facilities for disposal, and included training for diabetes clinic nurses and pharmacists in client counseling and waste handlers in the disposal of insulin syringe containers returned to facilities by clients. The Pilot found that, with training, nurses and pharmacists were able to counsel clients successfully. Insulin needle re-use declined from $87 \%$ to $8 \%$ with the provision of full supply and clients felt safer using a single needle for each injection, disposing used syringes into containers and returning full containers to facilities for disposal. All disposal containers worked well with no punctures or leakages, but used tablet containers and client sourced containers are the most sustainable in the Guyana context because they carry no additional cost and are readily available.
\end{abstract}

\section{Key Words}

Injection Safety, Insulin, Diabetes, Syringe Disposal, Syringe re-use, Guyana, Caribbean

\section{Corresponding author}

Rebecca Furth, Senior Technical Advisor, Initiatives Inc. 264 Beacon Street, 6th Floor, Boston, MA 02116.

Email: rfurth@initiativesinc.com, Tel: 617-262-0293, Fax: 617-262-2514, Web: www.initiativesinc.com 


\section{Introduction}

The safe use and disposal of injection equipment is a major component of infection control in clinical and community settings. Unsafe injections are estimated to account for 21 million new hepatitis B infections, 2 million hepatitis $\mathrm{C}$ infections and $2 \%$ of all new HIV infections worldwide each year. ${ }^{1,2}$ Safe needle use and disposal remain significant challenges in the developing world where resources are constrained and waste disposal systems are very limited or nonexistent.

Studies of injection safety typically focus on clinical settings where $95 \%$ of injections are provided, but issues of safety in non-clinical settings are also critical to community health and safety. Diabetic insulin users represent a significant population of injection equipment consumers whose practices have implications for community safety. Most insulin users inject at home; yet even in developed countries, there are few options for safely disposing of needles or lancets in the home setting. ${ }^{3} 4$ Furthermore, many insulin users prefer or require the assistance of a friend or family member to inject their insulin or dispose of needles, placing these helpers at risk of needle stick injuries that could expose them to blood borne diseases. ${ }^{5}$

Insulin needle reuse is common among persons with diabetes, this is especially true in resource poor countries where public facilities that serve a majority of clients with diabetes typically provide only a small number of needles required by each diabetic insulin user and where clients often do not have the financial resources to supplement the supplies they receive free of charge. ${ }^{6}$ The American Diabetes Association and International Diabetes Federation recommend single needle use for insulin injections, but acknowledge the practice of and provide guidelines for reuse. ${ }^{7,8} \mathrm{~A}$ recent case of improper use of insulin pens at a hospital in the United States, which is suspected to have contributed to Hepatitis $C$ infection in 16 individuals, underscores the potential for transmission of blood borne diseases through insulin injections. ${ }^{9,} 10$

In 2007, the USAID-funded Guyana Safer Injection Project (GSIP) and the Guyana Ministry of Health $(\mathrm{MOH})$ conducted an assessment to better understand insulin needle use and disposal practices. ${ }^{5}$ Based on the findings of the assessment, in 2008 GSIP and $\mathrm{MOH}$ developed and implemented a pilot program to improve the safety of needle use and disposal among insulin users injecting at home. The results of the pilot, which are reported in this paper, suggest that low cost, practical options for safe insulin needle use and disposal are possible in resource constrained countries. The provision of disposal containers and return of used needles to facilities for disposal are important measures for increasing the safety of insulin injection at home and reducing community risk of exposure to needle stick injuries and other dangers associated with sharps waste.

\section{The Guyana Context}

Although geographically part of South America, Guyana is culturally and politically Caribbean. More than $90 \%$ of the population of 766,000 lives in the six coastal regions, with $41 \%$ living in the capital region alone. Because much of the coastal area is virtually at sea level, neighborhoods are typically crisscrossed by canals to control water flow and flooding. Only a few major towns have municipal waste pick-up and disposal; most residents bury or burn trash in their yards, throw materials into latrine pits or dump into the canals. A majority of the population seeks heath services through the public sector. Persons with diabetes can be served at public health clinics, but those who require insulin are usually cared for at public hospitals, which are staffed by doctors. The Ministry of Health provides insulin and insulin syringes free of charge at public facilities.

There are an estimated 37,600 persons with diabetes in Guyana. ${ }^{11}$ The International Diabetes Federation estimates diabetes prevalence for 2010 at $9.5 \%$; a number expected to grow to $12 \%$ by $2030 .{ }^{12}$ No population-based data are available on insulin use for Guyana, but data gathered from three large government hospitals by GSIP suggest that an average of $35 \%$ of persons with diabetes who are undergoing treatment inject insulin at least one time a day, amounting to as many as 13,160 individuals. This is a relatively high percentage by global standards, but not unprecedented. The Diabetes Atlas notes that in studies documenting insulin use among persons with Type 2 diabetes, "the median use was $20 \%$ and ranged from $1 \%$ (inpatients, Argentina) to $40 \%$ (outpatient clinic, Mexico) ${ }^{\prime \prime} .^{13}$ 


\section{Assessing insulin needle use and disposal practices} GSIP and $\mathrm{MOH}$ designed the pilot program based on an assessment conducted in 2007 that evaluated the availability and use of oral diabetes medications and insulin, the existence and application of insulin prescription and injection protocols, and insulin needle storage and disposal methods. The assessment was conducted at three large public hospitals with select data collected from the Guyana Diabetics Association (GDA) and a private hospital (Table I). While this paper focuses on the findings of the pilot program, the results of the assessment provide an important foundation for understanding the context and the rationale for the pilot design and are, therefore, presented here in brief.

\section{Assessment Methods}

The assessment used interviews of physicians, nurses, pharmacists and waste handlers to learn about the availability and use of protocols and typical counseling practices. Record reviews of pharmacy and clinic records were conducted at each site to assess patient load, numbers receiving insulin and stocks of diabetes medications, record keeping practices, storage and dispensing of insulin and insulin syringes. To complement these clinic-based data, client home visits were conducted to better understand the practices and challenges clients face in using insulin and storing and disposing of insulin needles at home.

\section{Assessment Findings}

The assessment found that no protocols existed for insulin prescription, safe insulin needle use or insulin needle disposal. The Ministry of Health had drafted National Guidelines for Control and Management of Type 2 Diabetes, ${ }^{14}$ but these had not been finalized or disseminated at the time of the assessment and did not contain guidelines on safe needle use or disposal. Record reviews conducted during the course of the assessment indicated a wide range of insulin use among clients frequenting the different facilities with one government hospital having as many as $48 \%$ of its clients with diabetes on insulin, a second with $35 \%$ and a third with only $22 \%$. The lack of clear insulin prescription protocols likely contributed to this diversity in practice, but social, cultural and behavior practices may also be involved.

Providers at all sites offered some counseling on proper insulin use and injection, but little or no counseling on safe insulin needle use and disposal. $82 \%$ of the 39 clients interviewed reported re-using insulin needles, with an average needle use frequency of 3.3 times. $78 \%$ of clients who reported re-use said they could not obtain enough needles from the hospital to use once and 59\% stated that they could not afford a new needle for each injection.

Table I: Assessment data collection sites and methods

\begin{tabular}{lcccccc} 
Site/Instrument & $\begin{array}{c}\text { Client } \\
\text { interview }\end{array}$ & $\begin{array}{c}\text { Client } \\
\text { home visit }\end{array}$ & $\begin{array}{c}\text { Physician } \\
\text { interview }\end{array}$ & $\begin{array}{c}\text { Nurse } \\
\text { interview }\end{array}$ & $\begin{array}{c}\text { Pharmacist } \\
\text { interview }\end{array}$ & $\begin{array}{r}\text { Record } \\
\text { review }\end{array}$ \\
\hline Site 1 & 14 & 6 & 4 & 2 & 1 & 1 \\
\hline Site 2 & 10 & 6 & 2 & 2 & 1 & 1 \\
\hline Site 3 & $11 \mathrm{a}$ & 6 & 1 & 2 & 2 & 1 \\
\hline Site 4 & 0 & 0 & 2 & 2 & 1 & 0 \\
\hline GDA & 4 & 4 & NA & NA & NA & NA \\
\hline Total & 39 & 22 & 9 & 8 & 5 & 3 \\
\hline
\end{tabular}


All clients reported that their place of treatment was also their primary place to collect prescription medications. $82 \%$ of clients interviewed also stated that they primarily access insulin needles at the clinic site where they receive treatment.

$46 \%$ of clients interviewed reported receiving assistance with their insulin injections, while 33\% said that someone helps them to dispose of used needles. Assistance came almost exclusively from family members, primarily the client's daughter/daughterin-law or wife. Two clients reported knowledge of an accidental needle-stick injury having occurred, both in cases where a family member routinely assisted with the injections.

The assessment team conducted twenty-two home visits to learn more about insulin needle storage and disposal practices. Insulin users most commonly stored insulin syringes in the bedroom - often in a box or a plastic bag hung from a nail in the wall - or the kitchen, where they were frequently kept in the refrigerator with the insulin. In six homes new and used needles were kept together, underscoring the practice of needle re-use. While clients acknowledge concern about children tampering with their syringes, most locations were found to be accessible to children.

Insulin needle disposal methods reported were also unsafe. $56 \%$ of clients report disposing of used insulin needles in their household garbage to be collected by the municipality, while others burned them in household garbage pits, threw them in a canal or river, or threw them in a latrine. No clients interviewed reported taking used needles back to the treatment facility for disposal, and no facility reported having such a service available.

\section{Assessment Recommendations}

Based on the findings of the assessment, GSIP recommended testing safer use and disposal practices by trying:

1) provision of a full supply of insulin needles to each client for each prescription,

2) introduction of needle disposal containers which clients could use at home to store used needles safely,
3) initiation of a facility return program that would enable clients to return full containers to health facilities for disposal, and

4) improving client counseling by providers on safe needle use and disposal.

Protocols and guidelines for providers were also recommended as well as simple aids for clients to help communicate key messages.

\section{Pilot Testing Options}

\section{Methods}

GSIP and the $\mathrm{MOH}$ began testing out these approaches to improving the safety of insulin needle use and disposal in 2008. ${ }^{15}$ Five sites, three public hospitals and two private clinics, were selected to participate in the pilot based on the following criteria:

1) hospital sites with diabetes clinics,

2) diabetes clinics that see at least twenty-five clients per week,

3) diabetes clinics with one or more general practitioners on site,

4) hospitals with on-site pharmacies that dispense insulin, oral medications, and insulin needles/ syringes,

5) hospitals that are easily accessible.

To try and gain an understanding of how these approaches might work at the clinic level and in the private sector, two private clinics were also included in the pilot. The pilot program ran from January 15 to June 30, 2008 with some sites operating the program for longer than others due to logistical issues of getting disposal methods in country (Table II).

The pilot tested four methods of disposal: 2.5 liter safety boxes, a needle clipper, empty tablet containers sourced from hospital and clinic pharmacies, and client-sourced containers (Table III). To test out the cost and logistical implications of full needle supply, GSIP also provided the four hospital sites with enough syringes to supply each insulin user with a single syringe for each injection. Depending on the structure of service delivery at the site, providers or pharmacists used routine diabetes clinic visits to issue clients containers and syringes or instructed them on how to 
Table II: Sites and Clients

\begin{tabular}{lccc} 
Site & Site Type & Months Pilot & $\begin{array}{c}\text { Average number of insulin- } \\
\text { using diabetics seen per month }\end{array}$ \\
\hline Site 1 & Public Hospital & 4 & 209 \\
\hline Site 2 & Public Hospital & 2 & 114 \\
\hline Site 3 & Public Hospital & 3 & 33 \\
\hline Site 4 & Private Clinic & 6 & 3 \\
\hline Site 5 & Private Clinic & 6 & 6 \\
\hline
\end{tabular}

source containers. They advised clients to return the containers to the clinic at the next monthly visit or sooner if the container reached $3 / 4$ capacity before the next clinic date.

In the original design, Site 1 was to provide only empty tablet containers to clients. However, changes in drug ordering altered the supply of empty tablet containers. This mainly entailed containers coming from different manufacturers with different packaging practices resulting in smaller containers - suitable containers had to hold a minimum of 32 insulin syringes. As a result, the hospital experienced shortages of suitable tablet containers for several weeks. To ensure that clients could still safely store used syringes and return them to the hospital for disposal, GSIP provided guidance to clinic nurses on how to counsel clients to select appropriate disposal containers and a flyer was developed for distribution to clients to help remind them of key criteria in selecting a disposal container.

GSIP and $\mathrm{MOH}$ provided training to diabetes clinic nurses and pharmacists in counseling clients on safer insulin needle use and disposal practices; sourcing, stocking and ordering insulin syringes; receiving and storing full containers; and record keeping on syringe and disposal container distribution and return. Waste handlers were provided training in where and how to collect full containers. Physicians were invited to a one hour briefing on the program to make them aware of its existence, objectives and basic messages for clients on safe needle use and disposal.
At each site, syringe and disposal container distribution was integrated into existing systems. At Site 1, diabetes clinic nurses distributed syringes and disposal containers while the pharmacy distributed insulin (Table IV). Clinic nurses at Site 1 collected full disposal containers because the pharmacy cannot take in biological waste. At Site 2, the central pharmacy took responsibility for the distribution of syringes, insulin, and disposal containers and clinic nurses collected full disposal containers. At Site 3, the diabetes clinic pharmacy, which was separate from the main hospital pharmacy, distributed syringes and insulin while the clinic nurses distributed disposal containers and collected full containers from clients. Sites 4 and 5 were both very small clinics with one Medex playing multiple roles. At these sites, the Medex distributed syringes, insulin and disposal containers and collected returned containers.

Table III: Disposal Methods Tested by Site

Site Disposal method tested

Site $1 \quad$ Empty tablet containers and client sourced containers

Site 2.5 liter safety box

Site 3 Needle clipper and empty tablet containers

Site $4 \quad$ Empty tablet containers

Site $5 \quad$ Empty tablet containers 
Table IV: Syringe and Container Distribution Responsibilities

\begin{tabular}{lllll} 
Site & $\begin{array}{l}\text { Distributes } \\
\text { Syringes }\end{array}$ & $\begin{array}{l}\text { Distributes } \\
\text { Insulin }\end{array}$ & $\begin{array}{l}\text { Distributes Disposal } \\
\text { container }\end{array}$ & $\begin{array}{l}\text { Collects } \\
\text { returned containers }\end{array}$ \\
\hline Site 1 & $\begin{array}{l}\text { Diabetes Clinic } \\
\text { Nurses }\end{array}$ & Main Pharmacy & $\begin{array}{l}\text { Diabetes Clinic } \\
\text { Nurses }\end{array}$ & $\begin{array}{l}\text { Diabetes Clinic } \\
\text { Nurses }\end{array}$ \\
\hline Site 2 & Main Pharmacy & Main Pharmacy & Main Pharmacy & $\begin{array}{l}\text { Diabetes Clinic } \\
\text { Nurses }\end{array}$ \\
\hline Site 3 & $\begin{array}{l}\text { Diabetes Clinic } \\
\text { Pharmacy }\end{array}$ & $\begin{array}{l}\text { Diabetes Clinic } \\
\text { Pharmacy }\end{array}$ & $\begin{array}{l}\text { Diabetes Clinic } \\
\text { Nurses }\end{array}$ & $\begin{array}{l}\text { Diabetes Clinic } \\
\text { Nurses }\end{array}$ \\
\hline Site 4 & Medex & Medex & Medex & Medex \\
\hline Site 5 & Medex & Medex & Medex & Medex \\
\hline
\end{tabular}

GSIP conducted an evaluation of the pilot in July 2008 to assess the overall satisfaction of providers, waste handlers and clients with different aspects of the program, costs, safety and barriers to sustainability. The evaluation included analysis of data collected on numbers of clients counseled, syringes and disposal containers distributed and returned. It also included interviews with diabetes clinic nurses, pharmacists and waste handlers on satisfaction with the program, acceptability of any increased workload, challenges faced and suggested improvements. Additional client interviews were also conducted to gain a sense of client satisfaction, understanding of messages and experiences using and returning disposal containers. Finally costs of syringes and containers were assessed to gain a sense of long-term viability and sustainability.

\section{Results}

\section{Counseling}

The evaluation results showed that nurses and pharmacists provided counseling and that it was effective. This is significant in the Guyanese context were nurses do little counseling and it is generally believed that they will not do counseling or will not do it well if charged with the task. Nurses and pharmacists were willing to take on counseling tasks, were capable of providing quality counseling with the assistance of job aids, and did not find that counseling presented a significant work burden. $95 \%$ of the 75 clients interviewed said they received counseling on safe needle use and disposal. $97 \%$ said the nurse or pharmacist explained to them how to use the disposal container. $100 \%$ said the pharmacists explained why she was giving the specified number of needles. All $(100 \%)$ clients also reported receiving messages on needle disposal.

\section{Disposal Method Safety and Return}

All disposal containers tested were assessed to be safe. GSIP staff and regional consultants observed 77 disposal containers in client homes. Of these, 94\% were kept in places safe from child tampering and $96 \%$ were sufficiently isolated from other household objects. Only six containers $(8 \%)$ were observed to be more than $3 / 4$ full and none $(0 \%)$ were observed to have punctures, to be leaking fluid, or to be emitting offensive odors.

Return of containers to health facilities was, in general, better than expected. However, return to the largest hospital in the pilot program was significantly poorer than other sites (Table V). This hospital serves a large urban and peri-urban population some of which seeks services at other facilities, but comes to the hospital on a periodic basis. GSIP determined that a few clients had returned containers to other facilities not included in the pilot, but this accounted for only a small number. Return improved significantly through the course of the pilot suggesting that counseling and client reminders might address the problem. Record keeping also improved throughout the course of the pilot, poor record keeping may have contributed to 
Table V: Container Return

\begin{tabular}{llllllll} 
Site & $\begin{array}{l}\text { Months } \\
\text { pilot in } \\
\text { operation }\end{array}$ & $\begin{array}{l}\text { Total } \\
\text { disposal } \\
\text { containers } \\
\text { distributed }\end{array}$ & $\begin{array}{l}\text { Monthly } \\
\text { average } \\
\text { client } \\
\text { visits } \\
\text { attended }\end{array}$ & $\begin{array}{l}\text { Number of } \\
\text { clients } \\
\text { instructed } \\
\text { to source } \\
\text { own container }\end{array}$ & $\begin{array}{l}\text { Containers } \\
\text { Returned }\end{array}$ & $\begin{array}{l}\text { Containers } \\
\text { Expected } \\
\text { (minus } \\
\text { of June }\end{array}$ & $\begin{array}{l}\text { Containers } \\
\text { unaccounted } \\
\text { for }\end{array}$ \\
\hline Site 1 & 4 & 904 & 226 & 38 & 394 & 742 & 348 \\
\hline Site 2 & 2 & 231 & 116 & 0 & 129 & 153 & 24 \\
\hline Site 3 & 3 & 82 & 27 & 8 & 91 & 58 & -33 \\
\hline Site 4 & 6 & 25 & 4 & 0 & 25 & 25 & 0 \\
\hline Site 5 & 6 & 22 & 4 & 0 & 21 & 22 & 1 \\
\hline TOTAL & 1264 & & 46 & 660 & 1000 & 340 \\
\hline
\end{tabular}

under-reporting of retuned containers, as it did to underreporting of container distribution in the first month of the program at Site 3.

\section{Client Satisfaction}

Across the board, clients were highly satisfied with the insulin-needle disposal systems, including containers and return procedures. Clients rated the usability of methods between 4.2 and 4.5 out of 5 .

\section{Syringe Supply and Re-use}

Clients appreciated receiving a syringe for each injection. All (100\%) of the 75 clients interviewed said they liked receiving a full supply of syringes. $51 \%$ of clients said they liked having a needle for each injection because it was less painful or caused less bruising. Others said they felt better protected from infection. The provision of a full supply of insulin needles greatly reduced re-use. Only six (8\%) clients interviewed said they had re-used an insulin needle in the last month. This is a significant decline from the pre-pilot Insulin Home-use Assessment which found that $82 \%$ of clients re-used insulin needles.

Cost

Empty tablet containers emerge as the preferred and most cost effective option, but the issue of shortages needs to be addressed. Table VI shows cost differences among different disposal container options.

Table VI: Container Costs

\begin{tabular}{lcccc} 
Method & Per Unit & $\begin{array}{c}\text { Units required } \\
\text { per client per year }\end{array}$ & $\begin{array}{c}\text { Estimated annual cost } \\
\text { per client per year }\end{array}$ & $\begin{array}{c}\text { Total estimated } \\
\text { Annual cost } \\
\text { to MOH }\end{array}$ \\
\hline Empty Tablet Container & $\$ 0$ & 12 & $\$ 0$ & $\$ 0$ \\
\hline $2.5 \mathrm{~L}$ Safety Box & $\$ .79$ & 12 & $\$ 9.48$ & $\$ 140,351$ \\
\hline Needle Clipper & $\$ 4.5$ & .33 & $\$ 1.45$ & $\$ 21,467$ \\
\hline Client-sourced container & $\$ 0$ & 12 & $\$ 0$ & $\$ 0$ \\
\hline
\end{tabular}


Providing a full supply of insulin needles to each diabetic client in the country obviously has associated costs. In Guyana it will require an additional \$158,414USD (Table VII) for 2009 alone. However, it is unlikely that the Ministry of Health would bear this full cost, since not all clients seek services at government facilities. Furthermore, $\mathrm{MOH}$ and service providers could control costs by investing in provider and client education and behavior change and enforcing adherence to treatment protocols to reduce insulin prescription where appropriate.

\section{Provider and Waste Handler Support}

All $(100 \%)$ of the twenty-five nurses, pharmacists and waste handlers interviewed felt the program for safer insulin disposal and needle use should continue.

\section{Discussion}

Based on these findings, GSIP and $\mathrm{MOH}$ determined that facility-based insulin needle return programs are feasible and should be continued at pilot sites and rolled out to other key sites. To ensure the sustainability and safety of the program for scale-up, the distribution of empty tablet containers to clients for safe insulin needle disposal were recommended as other methods were too expensive to be sustained and the quality of client sourced containers is harder to control. At all sites, guidelines for client-sourced containers and procedures for counseling clients ensure staff can provide appropriate guidance should tablet containers run short. Staff responsibilities for collecting, organizing and distributing containers should be documented and adequate staffs need to be made available at peek clinic hours to implement the program effectively.

The provision of a full supply of insulin syringes to each client was well received and appreciated by clients and providers. The $\mathrm{MOH}$ determined that it was capable of bearing the added cost and is committed to reducing re-use and its attendant pain, scarring and infection risks. Systems for providing full supply need to be accompanied by safe needle return to facilities to ensure that the volume of sharps in communities does not increase.

Client counseling is an essential component of safe needle use and disposal programs for insulin-users. Counseling does not add significantly to provider workload and, when appropriate training and job aids are provided, can be carried out to a high standard. The use of simple flyers with safe insulin needle use and disposal messages and with information on selfsourcing containers is effective and should be provided to all clients at each visit.

Table VII: Estimate of Insulin Syringe Costs for All Insulin Users in Guyana (USD)

\begin{tabular}{llllcc} 
Syringes Supply & Syringe & Cost per Unit & \multicolumn{1}{c}{$\begin{array}{c}\text { Average Cost } \\
\text { per Client per year }\end{array}$} & $\begin{array}{c}\text { Total estimated } \\
\text { Annual cost } \\
\text { to MOH }\end{array}$ \\
\hline Current & $15 /$ client/month & $\begin{array}{l}\text { MOH current } \\
\text { syringe }\end{array}$ & .055 & $\$ 9.90$ & $\$ 146,570$ \\
\hline 1 Syringe/injection & $44 /$ client/month & $\begin{array}{l}\text { MOH current } \\
\text { syringe }\end{array}$ & .055 & $\$ 20.60$ & $\$ 429,937$ \\
\hline 1 Syringe/injection & $44 /$ client/month & $\begin{array}{l}\text { Less* expensive } \\
\text { syringe }\end{array}$ & .039 & $\$ 29.04$ & $\$ 304,983$ \\
\hline Estimated Additional Annual Cost to MOH of Full Insulin Syringe Supply & & $\$ 158,414$ \\
\hline
\end{tabular}

* GSIP found that the syringe provided by the Ministry of Health was considerably more expensive than alternatives on the market and clients complained the syringe available was painful. $\mathrm{MOH}$ is looking into changing the insulin syringe it supplies. 
Finally, the report of pilot findings recommended that Ministry of Health expand efforts to promote diabetes control and enforce adherence to treatment protocols to reduce, to the greatest extent possible, the number of persons with diabetes who require insulin. Investments in nutrition education, exercise promotion, and diabetes awareness and management counseling, if they result in the reduction of the number of insulin users, can reduce the long-term costs of insulin and insulin syringe provision and decrease the number of syringes in households and the environment.

Following this pilot program, the Ministry of Health developed a safer insulin needle use and disposal policy. The policy includes providing a full supply of insulin syringes to each insulin using client and implementing empty tablet container distribution and facility-return for syringe disposal with a clientsourced container back-up system. The counseling guidelines for insulin needle use and disposal are in the process of being integrated into a revised version of the National Guidelines for Control and Management of Type 2 Diabetes. ${ }^{14}$ Finally, a plan for the scale-up of the safer insulin needle use and disposal pilot has been drafted. GSIP developed a training of trainers curriculum and, together with the Ministry of Health, has trained representatives of six regions to develop implementation plans and train providers, pharmacists and waste handlers to implement the program. Several regional and facility representatives have made visits to pilot sites to learn from their experience.

This pilot program shows that simple, low cost methods for ensuring safe insulin needle use and disposal are feasible in resource constrained settings. Facility return of used insulin needles works and keeps sharps out of communities. While Guyana determined that the use of empty tablet containers with a back-up of client sourced containers was most sustainable for its context, all disposal methods tested were effective and were liked by clients. Other disposal containers may be more appropriate in different country contexts.
Similarly, other countries may not be able to fully subsidize the cost of providing a full supply of insulin syringes to each client and practical solutions to controlling infection in these circumstances are still required.

\section{Acknowledgements}

This pilot program was carried out with the support and participation of the Guyana Ministry of Health through the USAID-funded Guyana Safer Injection Project implemented by Initiatives Inc. under Task Order No. GHS-I-02-03-00040-00. Dr. Leslie Ramsammy, Minister of Health, provided invaluable support for the assessment and pilot. We also thank the management and staff at pilot sites for their willingness and commitment to participate in the program.

\section{Declaration}

This material was presented at the American Public Health Association conference as a Poster Presentation on November 7, 2009.

\section{Reference}

1. WHO, 2006. Fact Sheet N 231, October 2006. World Health Organization. www.who.int/entity/mediacentre/factsheets/ fs231/en/ [Accessed: September 9, 2009.]

2. Miller MA and Pisani E. The Cost of Unsafe Injections. Bulletin of the World Health Organization 1999; 77: 808-811.

3. Crawshaw G, Irwin DJ, Button J. Disposal of syringes, needles and lancepts used by diabetes patients in North East Essex. Communicable Diseases and Public Health 2002; 5: 134137.

4. Bouhanick B, Hadjadj S, Weekers L. What do the needles, syringes, lancets and reagent strips of diabetic patients become in the absense of a common attitude? About 1070 questionnaires in diabetic clinics. Diabetes Metab 2000; 26: 288-293.

5. Furth R. Insulin Home-use Assessment Report. Guyana Safer Injection Project. Boston: Initiatives Inc., 2007. Produced by Initiatives Inc. for the United States Agency for International Development.

6. Gobte N, 2009. Injection safety campaign among diabetics, a challenge to infection prevention practitioners in developing countries. International Journal of Infection Control, September 2008. http://www.ijic.info/article/view/3039/2222 [Accessed: January 22, 2009.]

7. American Diabetes Association. Position Statement: Insulin Administration. Diabetes Care 2004; Supplement: S106-S109. 
8. IDF. Pharmacological Management of Insulin. Curriculum Model III-3: Insulin. s.I. : 2008: Section 3, part 2 of 3.

9. Caldwell Alicia A, 2009. 16 patients have hepatitis in Army needle scare. Associated Press, 11 March 2009. www.ap.org [Accessed: September 9, 2009.]

10. FDA, 2009. Insulin Pens: Risk of Transmission of Bloodborne Pathogens from Shared Use. Medwatch the FDA Safety Information and Adverse Event Reporting Program, March 19, 2009. United States Federal Drug Administration, Silver Spring, MD,. http://www.fda.gov/Safety/MedWatch/SafetyInformation/ SafetyAlertsforHumanMedicalProducts/ucm127783.htm [Accessed: September 14, 2009.].

11. IDF, 2007. Diabetes Atlas, 2007. The International Diabetes Federation, Brussels Belguim. http://www.eatlas.idf.org/ index8b98.html [Accessed: September 1, 2009.]
12. IDF, 2009. Diabetes Atlas Country Profiles, 2009. The International Diabetes Federation, Brussels, Belguim. http:// www.diabetesatlas.org/book/export/html/250 [Accessed: November 3, 2009]

13. IDF. Diabetes Atlas 2007. The International Diabetes Federation, Brussels, Belguim. http://www.eatlas.idf.org/ indexe83e.html [Accessed: September 1, 2009.]

14. Guyana Ministry Of Health. National Guidelines for Control and Management of Type 2 Diabetes: Edition 1. Georgetown, Guyana: The Government of Guyana Ministry of Health, January 2007.

15. Furth R. Insulin Needle Use and Disposal Pilot Evaluation Report. Boston: Initiatives Inc. 2008. Produced by Initiatives Inc. for the United States Agency for International Development. 